\title{
New Strategies and Simulation Tools to Optically Design a Field of Heliostats
}

\author{
Paola Sansoni, Franco Francini, Daniela Fontani, Elisa Sani, \\ Luca Mercatelli, and David Jafrancesco
}

CNR-INO National Institute of Optics, Largo E. Fermi 6, 50125 Firenze, Italy

Correspondence should be addressed to Paola Sansoni; paola.sansoni@ino.it

Received 30 October 2012; Accepted 1 February 2013

Academic Editor: Kalvala Srinivas Reddy

Copyright (C) 2013 Paola Sansoni et al. This is an open access article distributed under the Creative Commons Attribution License, which permits unrestricted use, distribution, and reproduction in any medium, provided the original work is properly cited.

A heliostats field is an array of mirrors concentrating the solar power on a receiver, typically placed on a tower. Our research experience in the optical design of heliostats fields for Concentrating Solar Power plants suggested using apposite simulation procedures, which were especially developed to simplify the reproduction of heliostats fields and to support in the analysis of the tower plant performances. Our most practically useful simulation tools are presented in this paper with exemplificative application results. The proposed strategies are addressed to solve specific problems and to evidence particularly crucial features. The main program facilitates the heliostats field analysis, exploiting the integration between our software code and Zemax. A side program permits to assess effects of seasonal and daily variations of solar irradiation. Another dedicated side code simplifies the selection of a Compound Parabolic Concentrator as secondary optics.

\section{Introduction}

The paper proposes new simulation tools especially studied by our research group to facilitate the optical design of heliostats fields, which are typically employed in thermodynamic tower plants. Heliostats tower systems are Concentrating Solar Power (CSP) installations with principal components a heliostats field and a tower receiver. A heliostats field is an array of mirrors concentrating the solar power on a receiver, typically placed on a tower. The experience of our research group in the optical design of heliostats fields for CSP plants suggested developing specific software programs that can be used as assisting tools to facilitate the simulation of a multimirror field. This paper discusses applications and capabilities of our most practically useful tools. Their main purposes are to simplify the optical design of multimirror arrays and to support in the analysis of the solar plant performances.

Considering the optical point of view, for planning and simulating a multimirror plant it is necessary to use an optical design software package because it takes into account not only the interaction between luminous radiation and surfaces, the effects due to the whole solar spectrum, but also the effects due to solar divergence that in this case are significant because of the large distances involved. The software codes developed for our optical analyses offer the possibility to perform detailed and extensive investigations entailing specialised simulation programs. Unfortunately the case of tower plants involves an elevated amount of system components, so the manual insertion of all mirrors parameters can cause errors, and it is typically very tedious and time-consuming.

Some software codes, especially dedicated to the optical design of heliostat fields, were elaborated by other research groups for specific requirements, obtaining very promising results [1-4]. Two examples of these codes are described in Erminia Leonardi's publications [1,2]. Besides, our European colleagues of the PROMES group, within the French CNRS, developed a software, denominated SOLFAST 4D [3]. The Laboratory "PROcédés, Matériaux et Energie Solaire" (PROMES) is a Research Unity of the CNRS (Centre National de la Recherche Scientifique) located at Font Romeu Odeillo, France.

Our research group preferred to use commercial optical design packages for being able to exploit all the potentialities of these software programs. The utilization of optical design software improves the simulation procedure versatility. The 
advantage is that the solar field, with its components, is seen as an element to be designed, whose development can utilize all the potentialities of these software packages, like automatic optimization and radiometric calculations. This strategy allowed us to obtain results with elevated graphic characteristics, high precision, and high reliability.

The aim of the proposed work was to simplify the analysis of the heliostats field behaviour, exploiting the possibility of interfacing optical design codes with programming systems, like Octave. Our integrated simulation tools were created for guiding the system analysis from the optical point of view, for managing the large data amount, and for extracting the essential parameters, with a particular attention to the quantities that are critical for the examined heliostats field.

The next sections propose three of our simulation tools: Simulation Tool_1 (ST_1) performs the optical design of a heliostats field; Simulation Tool_2 (ST_2) evaluates the power collected by the tower plant; Simulation Tool_3 (ST_3) examines a polar conical surface as secondary optics.

An accurate design ( $S T_{-1}$ ) of the multimirror field involves various aspects, but it is essential for being capable of correctly assessing the power collected on the tower receiver by the heliostats (ST_2). Several features are important for our optical studies: first of all, the mirrors disposition must be defined to optimise the concentration of the light on the target $\left(S T_{-} 1\right)$. Beyond the receiver covering, there are other relevant aspects, like the study of seasonal and daily variations of solar irradiation $\left(S T \_2\right)$ on the tested CSP field, the shape and aperture of a possible CPC (Compound Parabolic Concentrator) receiver (ST_3), and the possibility to assess vignetting effects on the mirrors (ST 1 ).

The study of the interactions among solar rays, mirrors, and target is usually performed with the assistance of Zemax optical design software. The employment of this software package is justified owing to its capacity to manage and analyse a huge number of luminous rays, along with its possibility to generate irradiance maps (light distribution in $\mathrm{W} / \mathrm{m}^{2}$ unities) in predefined positions. The main application of the irradiance maps study is to analyse the behaviour of the radiation in proximity of the critical zones $\left(S T_{-} 1\right)$ : inside the target (a test surface placed in significant positions), within the furnace (the real receiving structure), and on the heliostats field. However the analysis of irradiance maps can also be useful to introduce potential modifications on the CSP plant geometry ( $S T_{-}$1). In practice, during the initial design phase, when the heliostats array geometry is defined, it is necessary to have the maximum freedom in the designation of the variables, taking into account that geometrical shape, azimuth, and elevation values must be identified for every mirror position. It is therefore essential to simplify the data insertion inside the software code $(S T 1)$, which should not be manually done because the multimirror field can include up to thousands of collectors.

\section{Simulation Tool_1: Optical Design of a Heliostats Field}

Simulation tool_1 (ST_1) represents the main proposed code, and it was created with the aim of facilitating the insertion of all the data pertaining to the multimirror tower plant, for being simulated in the optical design software package. We developed a software program, in Octave type language, which staring from the description of the CSP field is capable of interacting with Zemax to obtain an analysis of the beam distribution on the target.

Its application can be summarized as follows: ST_1 prepares a file containing all the geometrical parameters of the mirrors and their positioning on the field; successively the file could be read by a Zemax macroinstruction that will automatically import all the optical components.

In practice the program accepts the following inputs:

(i) geometrical position of the target;

(ii) azimuth and elevation of the sun;

(iii) positions of all mirrors composing the heliostat field, taken from a text format file;

(iv) in alternation to the previous point it is possible to prepare a field with predefined dimensions, as an $N \times$ $M$ heliostats matrix, oriented at a defined angle with respect to the tower.

The following parameters are determined for every mirror:

(i) $x, z$, and $y$ coordinates with respect to the tower, which is identified as axes origin;

(ii) curvature radius as a function of furnace entrance position;

(iii) local azimuth and elevation of every mirror to deviate the solar rays on the furnace in dependence of sun's azimuth and elevation;

(iv) besides it is possible to insert an "angular noise" on the mirror position in order to simulate a tracking error.

The data contained in the matrix of $N \times M$ mirrors are transferred in automatic modality, and a Zemax program supplies the following results:

(i) irradiance map on a suitable target plane;

(ii) map of the solar irradiance on the plane located under the heliostats, to evaluate the influence of the tower shadow and of the relative mirrors shadows (vignetting effects).

In particular it is useful to remember that every programme that uses ray-tracing techniques automatically takes into account the cosine effect on the mirrors. This means that the software code considers the mutual shadows between the heliostats rows and the consequent effect of sunlight block that a heliostats line can cause on the next mirrors row. The results of the calculation are therefore very precise, and they can successfully simulate the real plant conditions.

Exemplificative results of the optical simulation of a multimirror tower plant are presented in Figures 1-3. Each heliostats plant simulation is obtained inserting in Zemax the data pertaining to the position of mirrors, through the Octave interface of $S T$ 1. Then the Zemax software package 


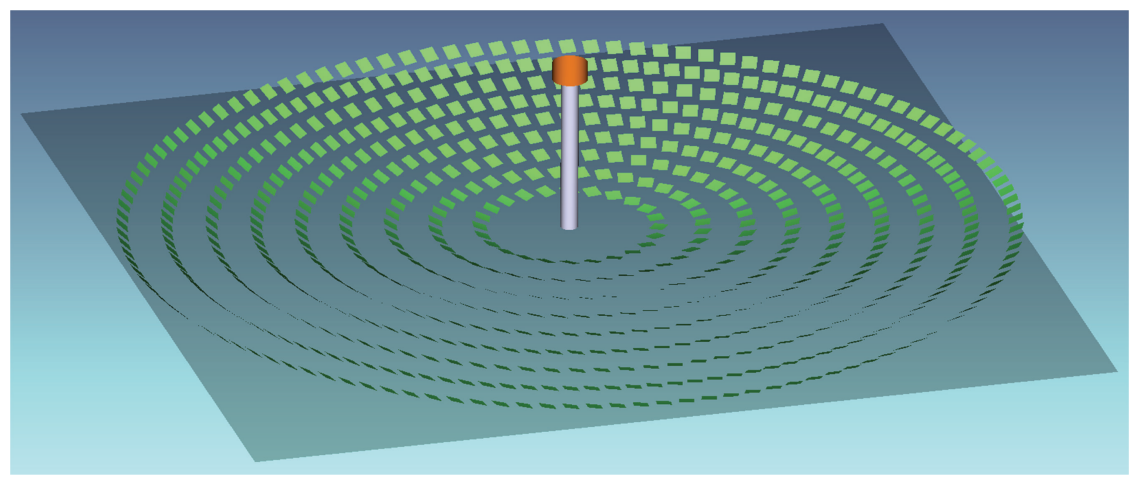

FIgURE 1: A 3D rendering of a multimirror tower plant.

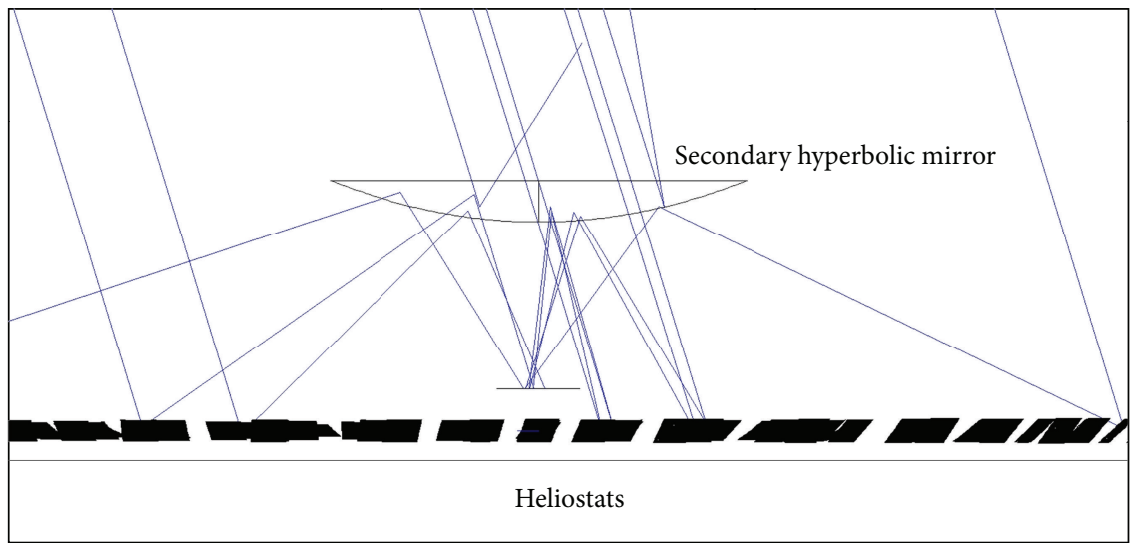

FIGURE 2: Scheme of a heliostats field of beam-down type, evidencing the rays reflected by the secondary hyperbolic mirror.

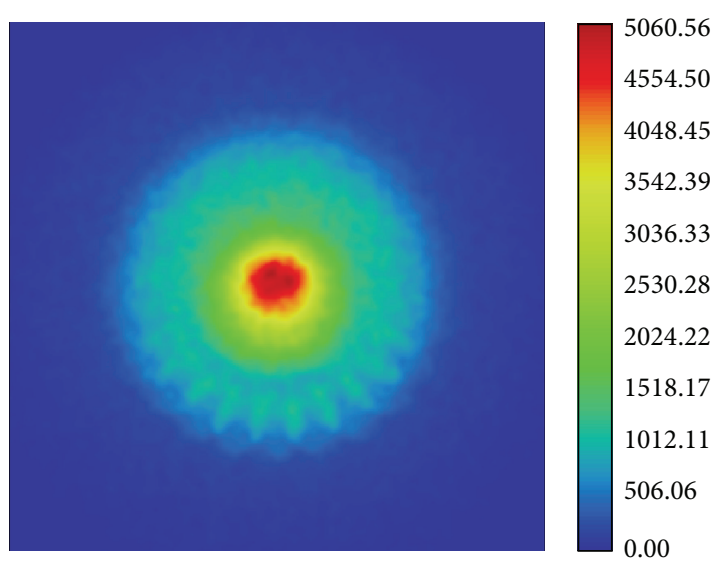

FIGURE 3: Example of a target irradiance map on a heliostat field.

can provide a 3D rendering of the solar plant, like Figure 1, a heliostats field scheme, like Figure 2, and irradiations maps, like the example shown in Figure 3. In the side view of Figure 2 the heliostats appear as dark quadrangles (often superimposed) in a bottom line, while the secondary optics is clearly visible at figure centre. The target irradiation map (in $\mathrm{W} / \mathrm{m}^{2}$ ) in Figure 3 presents the distribution over the tower target of the light concentrated by the array of mirrors composing the CSP plant.

In addition to the optical design of a multimirror field, which is the principal function of $S T \_1$, our main code allows additional functions: the first quantifies the effects of angular noise, while the second examines mutual shadows between mirrors.

In order to obtain a more realistic simulation of the actual practical realisation of the solar field, our program $S T$ I allows the insertion of an "angular noise." In particular, a Gaussian angular noise can be introduced on every mirror to simulate the effect of random disturbances on the position of the collectors. The application of a Gaussian angular noise on the heliostats orientation consists in adding this angular noise to the azimuth and elevation values of each mirror.

These errors are actually due to several different causes, like the imperfect positioning of a real mirror, tracking errors, wind effects, and so forth. The code of $S T \_1$ permits to set the variance value of the Gaussian noise, in order to analyse how much an incorrect orientation of the mirrors affects the illumination on the target. An example of application of the Gaussian angular noise on the mirror orientations is numerically reported in Table 1 and graphically visualized in Figures 4 and 5. In particular Figure 4 presents the Gaussian angular noise, corresponding to the angular deviation of mirrors orientation, for a Gaussian distribution with variance 0.011 . 
TABLE 1: Mirror angular noise, as a function of the Gaussian noise variance, and effect on the furnace illumination.

\begin{tabular}{lcc}
\hline $\begin{array}{l}\text { Variance of the Gaussian } \\
\text { angular noise }\end{array}$ & $\begin{array}{c}\text { FWHM noise } \\
\text { on the mirrors }\end{array}$ & $\begin{array}{c}\text { FWHM error } \\
\text { on the furnace }\end{array}$ \\
\hline 0.00625 & $\pm 0,093^{\circ}$ & $\pm 0,186^{\circ}$ \\
0.011 & $\pm 0,123^{\circ}$ & $\pm 0,247^{\circ}$ \\
0.0125 & $\pm 0,132^{\circ}$ & $\pm 0,263^{\circ}$ \\
0.025 & $\pm 0,186^{\circ}$ & $\pm 0,372^{\circ}$ \\
0.05 & $\pm 0,263^{\circ}$ & $\pm 0,527^{\circ}$ \\
\hline
\end{tabular}

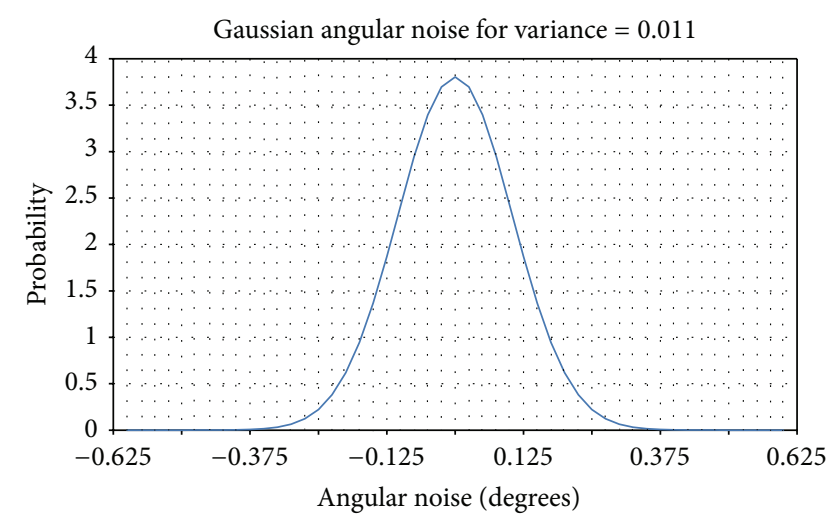

FIGURE 4: Angular deviation of mirrors orientation for a Gaussian distribution with variance 0.011 .

Then Figure 5 illustrates the effect of the Gaussian angular noise on the perfect target image shown in Figure 3.

Table 1 presents the noise of mirror orientation and the consequent error on the target for different values of variance of the Gaussian angular noise. Columns 2 and 3 report the FWHM (full width at half maximum) of the angular deviation, in a Gaussian distribution statistic. Column 2 refers to the angular deviation on the mirrors orientation (azimuth and elevation), while Column 3 reports the angular deviation of the ray impinging on the furnace target. Because of the specular reflection, the values in Column 3 (target angular error) are double than those in Column 2 (mirror angular noise).

These design considerations can be practically advantageous, since they give indications about the robustness or sensitivity of our optical design with respect to angular variations.

The possible shadows between mirror rows, causing vignetting effects, are easily observable using a function that is available in the $S T \_1$ code. A detector, located under the heliostats plane and having the same dimension of the multimirror field, visualises the shadows that the sun generates between the collectors rings. Figure 6 presents an example of visualisation of this vignetting effect. If the image presents superimposed shadows, it is evident that the solar rays cannot completely illuminate all mirrors, therefore generating even significant reductions of the plant performances. For a correct optical design of the solar plant the vignetting effect simulation should be repeated for various

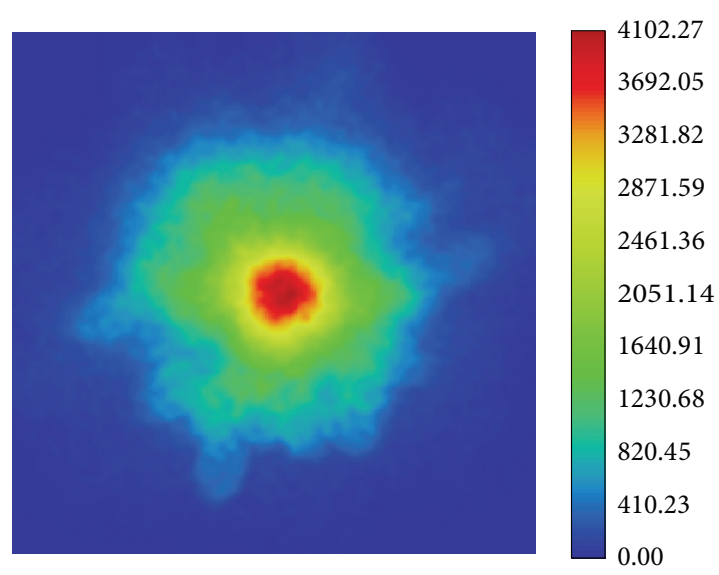

FIGURE 5: The target irradiance map of Figure 3 with the effect of a Gaussian angular noise.

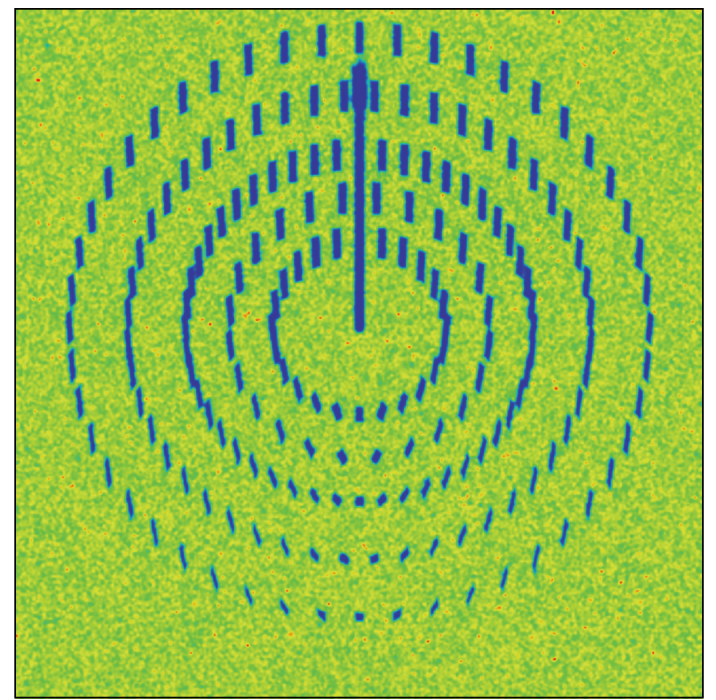

FIGURE 6: Example of the shadows of mirrors and tower in a circular field.

hours of the day, in order to optimise the multimirror array design for the whole working period.

\section{Simulation Tool_2: Evaluation of the Power Collected by the Field}

Another fundamental aspect in the analysis of heliostats plants is the capacity of the CSP field to collect solar energy. A dedicated program, indicated as Simulation Tool 2 (ST_2), was developed for examining this multimirror field characteristic. This program, starting from the heliostats field geometry, calculates the solar power on each mirror composing the solar field. ST 2 code permits to precisely evaluate the quantity of energy collected by the tower plant during the day and for each single day of the year. Conversely to $S T \perp$, $S T \_2$ does not require the use of Zemax, and it is faster than $S T \_1$ in the calculation of the results. 


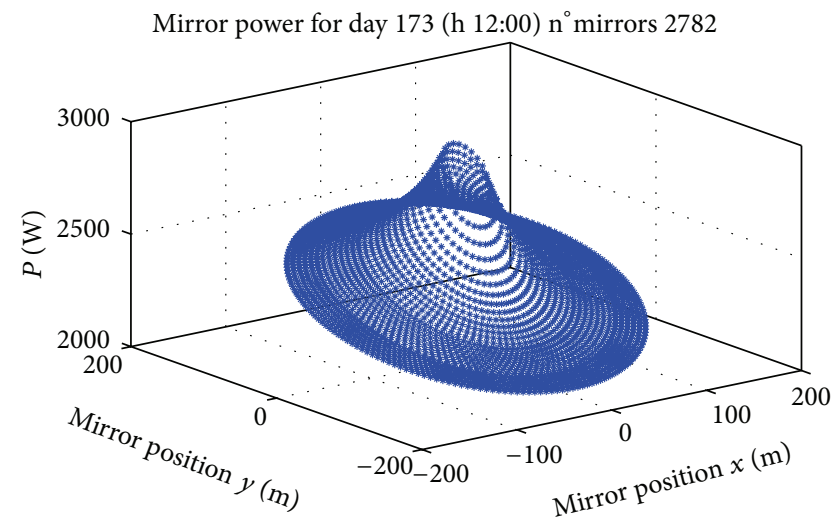

Figure 7: A 3 D view of the power $P$ collected by each mirror $\left(3 \mathrm{~m}^{2}\right)$ in the solar field.

This type of evaluation (ST_2) is of extreme importance in the phase of optical design of a heliostats field, because it allows verifying performances and efficiency levels of the solar plant as a function of mirrors field geometry and furnace position.

The solar irradiance data $I_{s}$ must be introduced as input data in Simulation Tool_2. However ST_2 includes an alternative automatic modality for the intensity calculation, as a function of the angle of sun's elevation, considering the effect of the air mass interposed between sun and heliostats. But the sunlight attenuation also depends on other parameters that are not easily predictable like water vapour content, aerosol, and pollution, which must be related with plant location, season, and atmospheric conditions [5]. Since it is impossible to take precisely into account all these aspects, we chose a simplified model that introduces an empirical coefficient $Q=$ 1.1 to take into account also the diffused component $[6,7]$, and the solar irradiance $I_{s}$ can be estimated as

$$
I_{s}=\mathrm{Q} \times I_{0} \times 0.7^{\left(A M^{0.678}\right)} \text {. }
$$

$I_{0}=1.353 \mathrm{~kW} / \mathrm{m}^{2}$ represents the solar irradiance outside the Earth atmosphere, and $A M$ is the Air Mass coefficient [8]. Considering that the field of heliostats reflects on the target only the direct radiation, the value of $Q$ must necessarily be set to 1 :

$$
A M=\frac{1}{\cos z+0.50572 \cdot(96.07995-z)^{-1.6364}},
$$

where $z$ represents the angle that the sun forms with the zenith (zenith angle).

The solar irradiance value $I_{r}$ reflected towards the tower by the single mirror is determined by the following equation:

$$
\begin{gathered}
I_{r}=\mathbf{S} \cdot \mathbf{U} \cdot I_{S} \cdot R \cdot A_{s p}, \\
\mathbf{S}=\left[\begin{array}{lll}
S_{x} & S_{y} & S_{z}
\end{array}\right], \\
\mathbf{U}=\left[\begin{array}{lll}
U_{x} & U_{y} & U_{z}
\end{array}\right],
\end{gathered}
$$

where $S_{x}, S_{y}$, and $S_{z}$ represent the unit vectors of the solar ray and $U_{x}, U_{y}$, and $U_{z}$ represent the unit vectors of the mirror

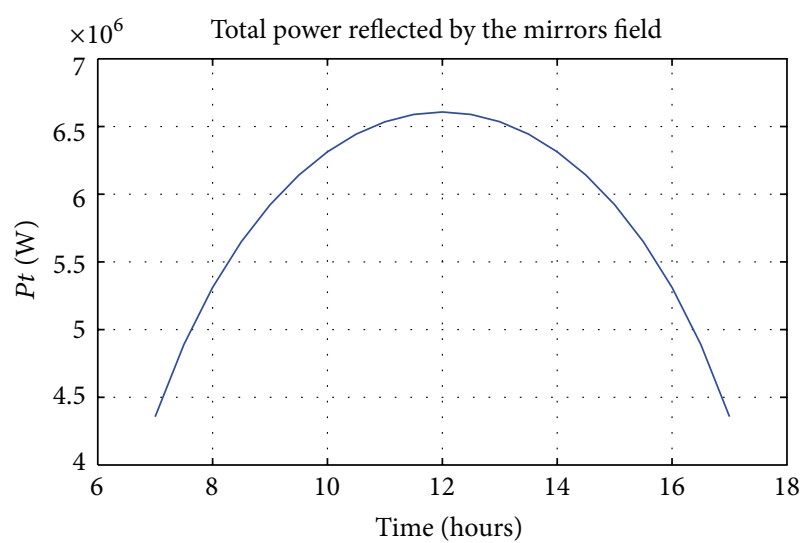

FIGURE 8: Total power reflected by the mirrors field at different hours of the day.

normal axis. Finally $R$ and $A_{s p}$ are reflectance and heliostat surface, respectively.

The main advantage of the application of ST_2 is to facilitate the definition of the initial geometry for the heliostats array and to permit the assessment of daily and seasonal fluctuations of collected power.

Examples of two types of results that can be obtained are presented in Figures 7 and 8. Figure 7 reports a 3D view of the power $P$ collected by each mirror in the solar field, where the area of every mirror is $3 \mathrm{~m}^{2}$. Figure 8 presents the total power $P t$ reflected by the mirrors field toward the target at different hours of the day.

Besides it is possible to visualise the received solar power behaviour in a movie format.

\section{Simulation Tool_3: Polar Conical Surface as Secondary Optics}

The last software tool proposed in this paper refers to the utilisation of a secondary optics in the furnace: in particular Simulation Tool_3 (ST_3) allows introducing a polar conical surface as secondary optics of the collection system.

The angular aperture $\theta$ of a possible secondary concentrator must be defined considering the position of all mirrors composing the solar tower plant. Consequently the value of $\theta$ affects the geometry of the multimirror field, and, conversely, the plant geometry affects the angular aperture of the secondary optics. A frequently employed secondary optics is the Compound Parabolic Concentrator (CPC).

$S T \_3$ program allows simulating the field of view $\theta$ of a $C P C$ to simplify the operations of optical design of heliostats fields. In this case the concentrator is no more considered as a receiver, but as a luminous source that projects a luminous cone on the ground. The interception between the cone and the ground defines the limitations that should contain the mirrors of the heliostats array.

Figure 9 presents an example of a calculation for a $C P C$ placed at a height $(h)$ of $25 \mathrm{~m}$, at a horizontal coordinate (dist) of $20 \mathrm{~m}$, with an inclination angle (Alfa) of $51.3^{\circ}$ (of the cone axis with respect to the ground). The tower is 


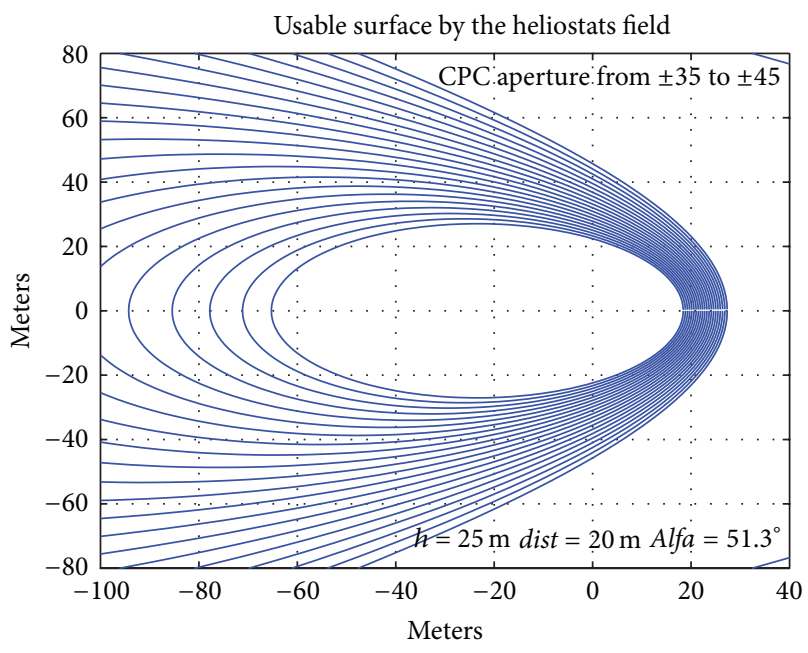

FIGURE 9: Useful surface in the heliostats field as a function of angular aperture and orientation of the CPC secondary optics.

located in position $(20 \mathrm{~m} ; 0)$ of the chosen coordinates. Every possible $C P C$ aperture corresponds to a curve on the plot of Figure 9. The range for the total angular aperture $\theta$ of the $C P C$ is between 70 degrees $\left( \pm 35^{\circ}\right)$ and 90 degrees $\left( \pm 45^{\circ}\right)$. The aperture of the Compound Parabolic Concentrator, selected as secondary optics, must be chosen in order that the mirrors of the solar plant are contained inside the corresponding area.

\section{Conclusion}

Other research groups chose to construct a software program dedicated to the optical design of heliostats fields, obtaining very good results. Our research group preferred to use commercial optical design packages for being able to exploit all the potentialities of these software programs. This strategy allowed us to utilise optimisation processes and radiometric calculations, finally obtaining results with elevated precision, advanced graphic characteristics, and high reliability.

The proposed simulation instruments are practically useful for developing optical designs of heliostats tower plants. Being derived from our experience in the research on solar plant design, they are addressed to solve specific problems and to evidence particularly crucial features. This paper presented the most advantageous tools, among our simulation programs for heliostats field design, and their practical applications were discussed showing examples of results.

The main code ST_1 performs several functions: it provides a rapid and easy-to-manage optical design of a heliostats field; it gives the irradiance map on a target of the heliostats field; it estimates the effect of a Gaussian angular noise on the heliostats positions; it assesses the possible shadows (vignetting effect) between mirror rows. The aim of $S T 1$ code is to facilitate the heliostats field analysis, exploiting the integration between our simulation code and ray-tracing software packages (Zemax). It simplifies optical design and ray-tracing simulation of the multimirror array, representing the key component of every thermodynamic tower plant. The heliostats field should be precisely shaped, placed, and oriented to concentrate the sunlight on the tower receiver. The collection performances of the solar plant should be examined considering the solar variations during the day and during the year, finally selecting the optimal configuration.

The two side programs are more specific: $S T \_2$ evaluates the power collected by the heliostats field; $S T \_3$, using a polar conical surface (a $C P C$ ) as secondary optics, selects the $C P C$ aperture. The side-program ST 2 permits to assess effects of seasonal and daily variations of solar irradiation. The dedicated side-code ST_3 simplifies the selection of a Compound Parabolic Concentrator (CPC) as secondary optics of the furnace.

Our simulation tools and optical design strategies were developed for the application to a precise thermodynamic tower plant simulation, which we can consider as reference case for our studies. In our reference heliostat field, the study of interactions between solar rays, mirrors, and target was carried out employing Zemax as optical design software package. The utilization of this simulation program is justified on the base of its capacity to analyse a great amount of luminous rays and its possibility to use these characteristics for simulating irradiance maps in proximity of the critical zones: in the test target, within the furnace and on the heliostats plant.

In order to simplify the analysis of the solar plant features, we exploited the possibility of interfacing our optical design packages with codes in Octave style. These integrated simulation tools were developed for guiding the optical system analysis, for managing the large data amount, and for extracting the major and crucial parameters of the multimirror field.

\section{Nomenclature}

Alfa: CPC inclination angle (between cone axis and ground)

AM: $\quad$ Air mass coefficient

$A_{s p}: \quad$ Heliostat surface

CPC: Compound parabolic concentrator

CSP: Concentrating solar power

dist: Horizontal coordinate at which the CPC is placed

$h: \quad$ Height at which the CPC is placed

$I_{0}$ : $\quad$ Solar irradiance outside the Earth

atmosphere

$I_{r}: \quad$ Solar irradiance reflected towards the

tower by the single mirror

$I_{s}: \quad$ Solar irradiance

$N \times M$ : Heliostats matrix

Octave: High-level programming language for numerical computations

$P$ : $\quad$ Power collected by each mirror in the solar field

Pt: $\quad$ Total power reflected by the mirrors field toward the target

Q: $\quad$ Empirical coefficient to take into account

also the diffused component

$R: \quad$ Reflectance

ST_1: Simulation Tool_1

ST_2: Simulation Tool_2

ST_3: Simulation Tool_3 
$S_{x}, S_{y}, S_{z}: \quad$ Unit vectors of the solar ray

$U_{x}, U_{y}, U_{z}$ : Unit vectors of the mirror normal axis

$x, z, y$ : Coordinates with respect to the tower (origin)

$z: \quad$ Angle that the sun forms with the zenith (zenith angle).

Zemax: Optical design software by ray tracing

$\theta$ : $\quad$ Field of view or total angular aperture of the CPC (as secondary collector).

\section{References}

[1] E. Leonardi, "Detailed analysis of the solar power collected in a beam-down central receiver system," Solar Energy, vol. 86, no. 2, pp. 734-745, 2012.

[2] E. Leonardi and B. D'Aguanno, "CRS4-2: a numerical code for the calculation of the solar power collected in a central receiver system," Energy, vol. 36, no. 8, pp. 4828-4837, 2011.

[3] "SOLFAST 4D by the PROMES Group-CNRS (French National Research Council)," http://www.hpc-sa.com/en/products/solfast4d.

[4] Tonatiuh 1.2.6, "Ray tracing for solar energy".

[5] V. Badescu, Ed., Modeling Solar Radiation at the Earth Surface, Springer, Berlin, Germany, 2008.

[6] A. B. Meinel and M. P. Meinel, Applied Solar Energy, Addison Wesley, New York, NY, USA, 1976.

[7] PVCDROM, Stuart Bowden and Christiana Honsberg, Solar Power Labs, Arizona State University, 2011.

[8] F. Kasten and A. T. Young, "Revised optical air mass tables and approximation formula," Applied Optics, vol. 28, no. 22, pp. 4735-4738, 1989. 

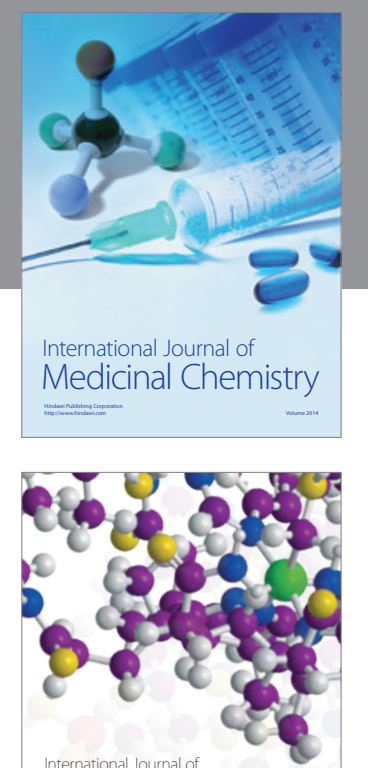

\section{Carbohydrate} Chemistry

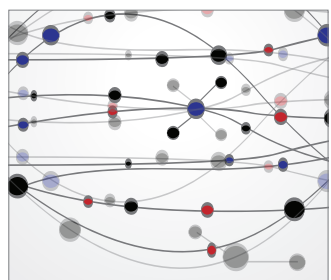

The Scientific World Journal
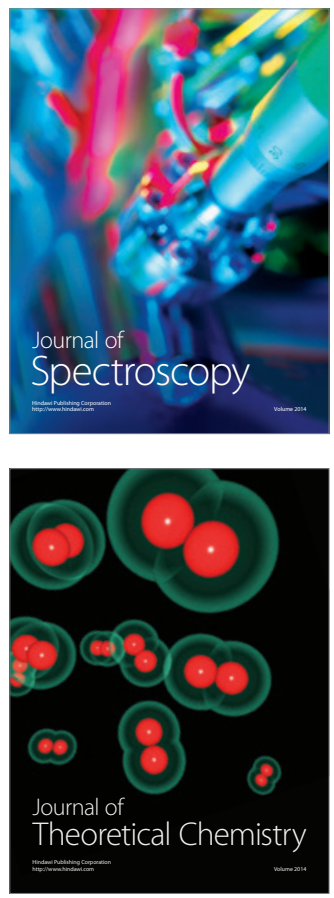
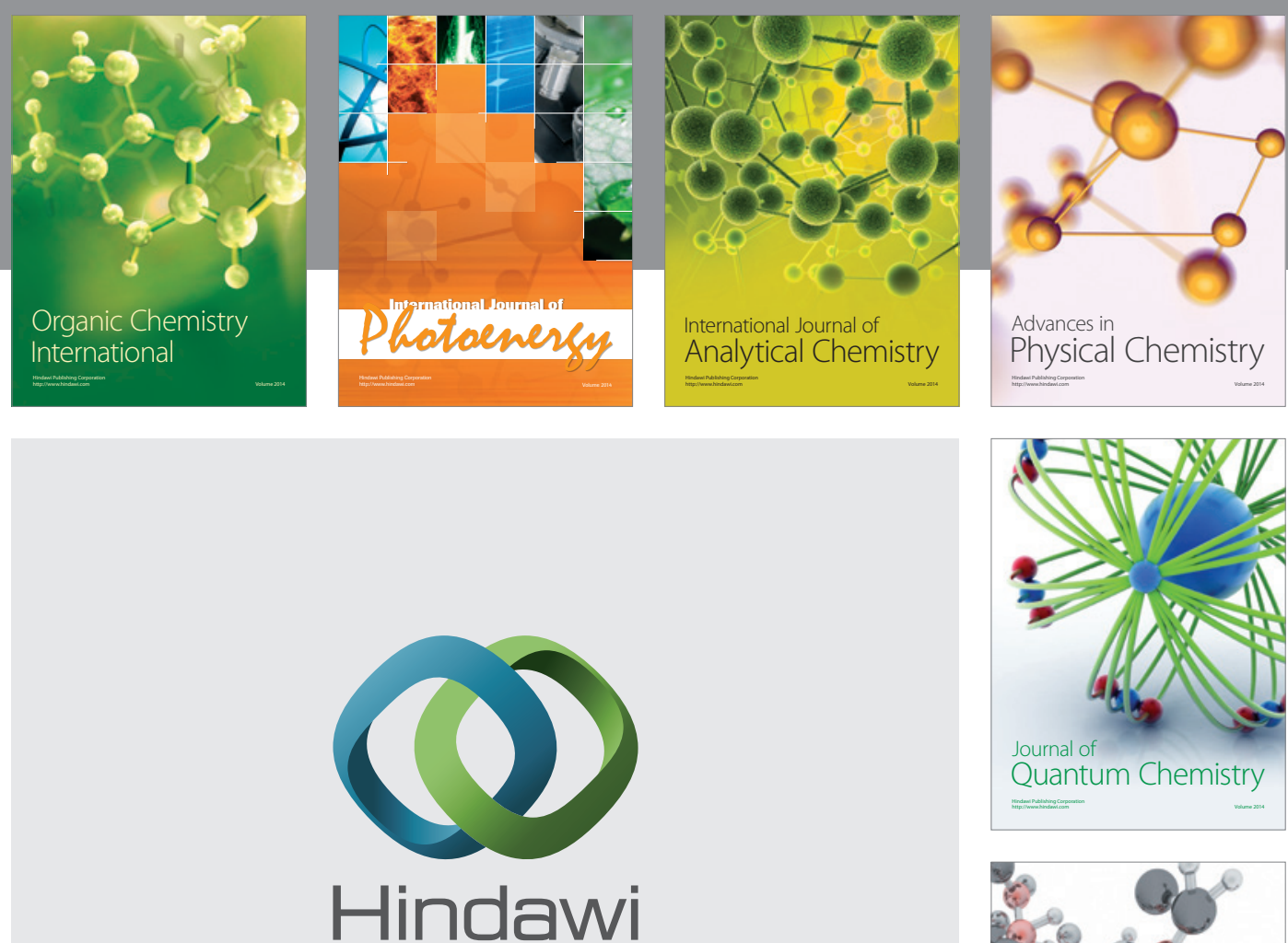

Submit your manuscripts at

http://www.hindawi.com

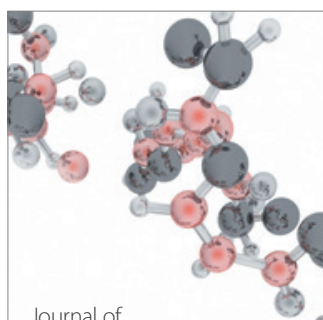

Analytical Methods

in Chemistry

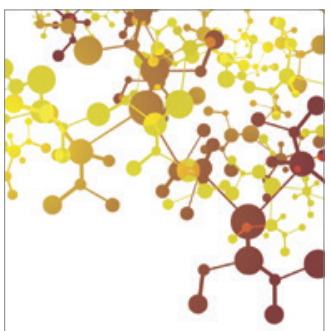

Journal of

Applied Chemistry

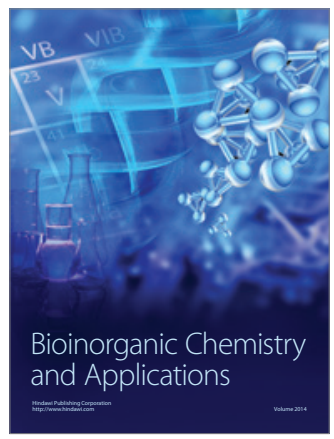

Inorganic Chemistry
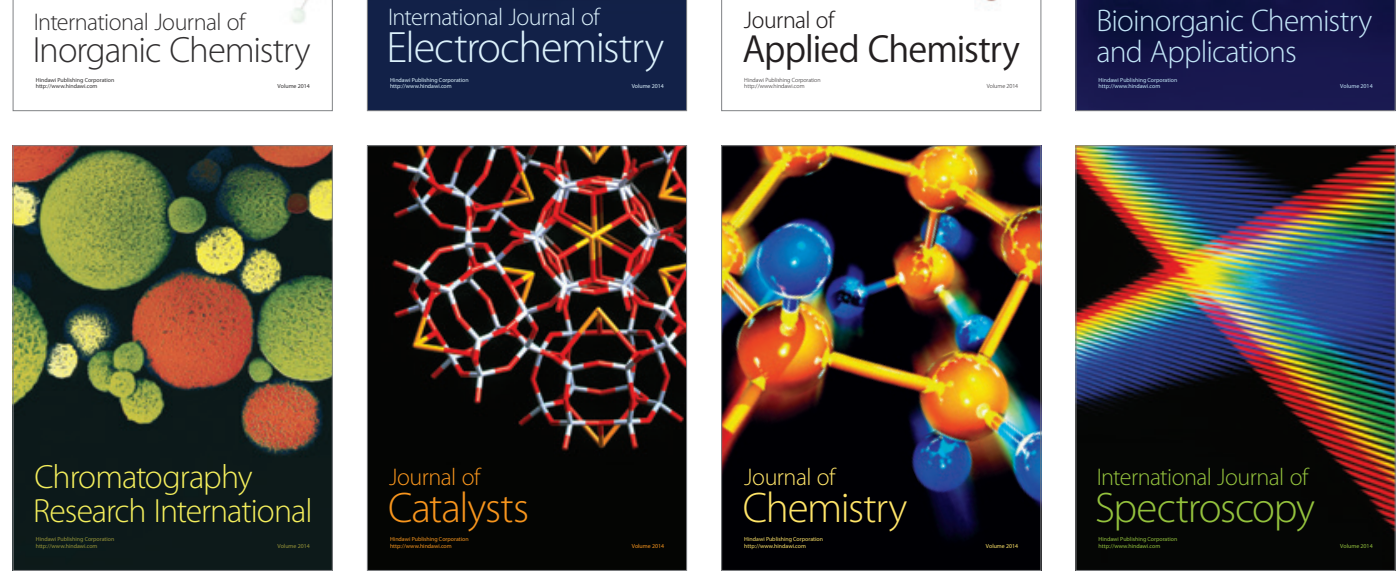